\title{
Psychological Impact of COVID-19 on Hispanic/Latine**, Asian, and White USA College Students
}

\author{
Lisa Mori $^{1 *}$, Kiran Kaur ${ }^{2}$, Maximiliano Gutierrez ${ }^{1}$, Valerie Klein ${ }^{1}$, Alan Guandique ${ }^{1}$ and Eric Cortez $^{1}$ \\ ${ }^{1}$ Department of Psychology, California State University, Fullerton, USA \\ ${ }^{2}$ Department of Psychology, University of Utah, USA \\ **Latine is a gender-neutral alternate term for Latinx that is easier to conjugate in Spanish and English.
}

"Corresponding author: Lisa Mori, PhD, Professor, Department of Psychology (H-830M), California State University, Fullerton, 800 N State College Blvd, Fullerton, CA 92831, USA, Tel: +16572783761; E-mail: lmori@ fullerton.edu

Received: November 27, 2020; Accepted: December 08, 2020; Published: December 16, 2020

\begin{abstract}
Background: Anxiety and stress among college students have sharply increased over the past two decades. COVID-19 exacerbated the mental health and academic challenges of United States (US) college students, particularly students of color.

Objective: To investigate the impact of COVID-19 on anxiety, stress and coping of Hispanic/Latine, Asian, and non-Hispanic White (NHW) US college students. Explore students' academic perceptions as predictors of anxiety and stress.

Methods: In this descriptive, cross-sectional study, 855 participants were students attending California State University, Fullerton (CSUF), a large public university in metropolitan Orange County, California, USA. From April to May 2020, participants answered an online survey of anxiety, stress, and coping. Demographic information and academic experiences were also collected online using a researcher-designed questionnaire. Frequencies and percentages summarized categorical data, and associations were analyzed using crosstabs, correlations, and multiple regression.

$\underline{\text { Results: }}$ Participants identifying as Hispanic/Latine $(n=449)$, Asian $(n=169)$, and non-Hispanic White $(\mathrm{NHW}$; $n=237)$ indicated difficulty transitioning to remote instruction and low academic engagement post-COVID-19. Over 70\% of students reported moderate to high anxiety, while $80+\%$ reported significant stress; these rates were typically higher for Asian and Hispanic/Latine participants than NHW participants. The leading healthy coping activity was exercise; the leading unhealthy coping behavior was alcohol abuse. Academic perceptions negatively predicted anxiety and stress levels.

Conclusion: COVID-19 adversely affected the psychological well-being of participants of color. Administrators should address students' mental health needs and provide culturally sensitive services. Additional remote instruction resources should be provided to these vulnerable students.
\end{abstract}

Keywords: Anxiety; COVID-19; Asian; Hispanic/Latine; College students

Citation: Mori L, Kaur K, Gutierrez M, et al. Psychological Impact of COVID-19 on Hispanic/Latine ${ }^{* *}$, Asian, and White USA College Students. J Anxiety Depress. 2020;3(2):128. 
www.yumedtext.com | December-2020 | ISSN: 2582-3264 | https://dx.doi.org/10.46527/2582-3264.128

\section{Introduction}

Prior to COVID-19, the mental health of US college students was a significant concern. Public health researcher Richard Scheffler described anxiety as being at epidemic levels among college students [1]. The 2018 National College Health Assessment [2] found that $62.3 \%$ of respondents reported "overwhelming anxiety", $41.4 \%$ felt "so depressed that it was difficult to function", and 85.5\% felt "overwhelmed by all (I) had to do" in the past year. The Healthy Minds Study 2018-2019 [3] indicated that $31 \%$ of college participants met criteria for Generalized Anxiety Disorder alone. According to the 2019 Annual Report of the Center for Collegiate Mental Health [4], anxiety, depression, and stress were the top three problems identified by students seeking psychotherapy through their universities. Students of color, LGBTQIA+, underrepresented groups, and lowincome students are particularly vulnerable to deleterious effects of stressful events such as COVID-19 and yet, are less likely to seek out mental health services than their White cisgender peers [5,6].

The global health crisis instigated by COVID-19 led to United States (US) government-mandated shelter-in-place orders in March of 2020. Nationwide, thousands of colleges, and universities abruptly switched to remote instruction, and the psychological impact of these changes on students quickly became a primary focus for administrators, health professionals, and faculty. Lederman [7] reported that over ninety percent of surveyed university presidents indicated that the mental health of students during the coronavirus pandemic was their highest concern.

Data provided by The Impact of COVID-19 on College Student Well-Being survey [8] demonstrated increased rates of anxiety, depression, and reduced psychological well-being, as well as greater impairment of academic performance due to mental health factors, pre- versus post-coronavirus pandemic mandated changes. In addition, about $60 \%$ of those college students who sought mental health treatment stated that access to such care was more difficult since the onset of the pandemic. Active Minds [9] found that over $80 \%$ of college students surveyed felt that their mental health was negatively affected by COVID-19, with 91\% reporting increased stress or anxiety. Likewise, Huckins et al. [10] reported an association between COVID-19 news and increased rates of anxiety and depression in their sample of US college students. Findings of a June 2020 survey by TimelyMD [11] demonstrated that coronavirus-related stress and anxiety among college students continued into the summer month of June when most students were not taking classes, with $85 \%$ of participants endorsing elevated levels of anxiety and stress. These findings for US college students are consistent with those of similar studies of college students around the world, such as in China [12], Indonesia [13], Spain [14], Israel [15], France [16], and Jordan [17].

\subsection{Statement of the problem}

In the US, the college population was described pre-COVID-19 as experiencing an "epidemic" of mental illnesses, chief among these anxiety, stress, and depressive disorders [1]. Underrepresented ethnic groups, such as African American/Black and Hispanic/Latine college students, as well as Asian collegiates, appear to be at even greater risk than their NHW counterparts of developing clinically significant anxiety, depression, and stress syndromes. For example, annual survey results of the National College Health Association [2] indicated that anxiety disorder rates between 2008 and 2016 rose 109\% for NHW students, $150 \%$ for Asian and Hispanic/Latine students, and 180\% for African American/Black students.

Asian and Hispanic/Latine represent the two fastest growing ethnic groups in the US [18]. Yet, there is limited research available about contributing factors to mental health problems and lack of accessing related services for college students hailing 
www.yumedtext.com | December-2020 | ISSN: 2582-3264 | https://dx.doi.org/10.46527/2582-3264.128

from these two groups. It is speculated that students of color face additional stressors than their NHW peers, such as racial/ethnic discrimination and stereotyping, and that these unique stressors may lead to greater vulnerability to anxiety and depressive symptoms. Heritage cultural views of psychological issues and treatment are also potential barriers to reporting and seeking treatment for mental health concerns.

With the additional stressors related to COVID-19, anxiety, depression, and stress disorder rates have increased for such students but they are less likely to report symptoms and are less apt to seek professional intervention than their NHW classmates. Organizations like Active Minds and the National College Health Association continue to conduct largescale surveys of the psychological toll of COVID-19 on college students. However, it is important to go beyond prevalence mental health surveys for vulnerable groups that underutilize psychological services, such as Hispanic/Latine and Asian college students, and identify specific COVID-19 predictors of anxiety and stress.

\subsection{Objectives}

The current study investigated the impact of COVID-19 on Asian, Hispanic/Latine, and NHW US college students' academic experiences, stress and anxiety levels, and coping. In California (the site of the current study), almost all public and private college classes were quickly transitioned to remote instruction in March 2020 and most were expected to remain predominantly online through the end of 2020 and beyond. This situation, along with several other COVID-19 changes, added to the already high stress and anxiety levels of California's ethnically diverse college population. Consequently, this investigation was intended to provide descriptive data on the academic perceptions, anxiety, stress, and coping after 6 to 8 weeks of COVID-19 restrictions in a sample of predominantly Hispanic/Latine college students. Additionally, academic perceptions were examined as predictors of anxiety and stress. Given that the sample was from a Hispanic serving institution, results may provide particular insight into how COVID-19 has affected Hispanic/Latine college students as well as their Asian and NHW peers.

\section{Methods}

\subsection{Participants}

Using variables such as race/ethnicity, gender, and academic designation, representative subgroups were created of all enrolled students $(\mathrm{N}=38,672)$ in the Spring 2020 semester at California State University, Fullerton (CSUF). From these subgroups, a random $20 \%(\mathrm{~N}=7,718)$ sample was generated. These students were emailed a request for voluntary participation in this survey (no material incentives were offered). The original sample contained 1,277 participants for a response rate of $16.55 \%$, which is within the typical response rate ranges of $5 \%$ to $30 \%$ for email survey solicitations [19]. Factors that may have contributed to the low response rate include the lack of extrinsic incentives and survey length in that surveys that take longer than 10 minutes to complete, like the survey used in the present study, are associated with response rate decreases as large as $40 \%$ compared to response rates for surveys that have brief completion times, such as 5 minutes or less [20]. Further, Fan and Yan $[21,22]$ estimated that the response rate for online surveys is $11 \%$ lower than that of other survey modalities. Incomplete surveys were excluded from the study; 268 participants were removed due to incomplete data. This sample consisted of 1,009 students (256 males, 736 females, and 16 other). The majority of the sample was composed of undergraduate (91.2\%) students, with $8.8 \%$ graduate students. A history of mental illness was reported by 322 participants (31.91\%). The most commonly reported diagnoses were major depressive disorder (MDD; 6.1\%), generalized anxiety disorder (GAD; 5.1\%), and post-traumatic stress disorder (PTSD; 1.1\%), and in combination (MDD and GAD: 8\%; MDD, GAD, PTSD: 1.8\%). 
www.yumedtext.com | December-2020 | ISSN: 2582-3264 | https://dx.doi.org/10.46527/2582-3264.128

Participants most commonly identified as Hispanic/Latine (44.5\%; $\mathrm{n}=449$ ), with $23.5 \%$ identifying as non-Hispanic White ( $\mathrm{n}=237), 16.7 \%$ as Asian ( $\mathrm{n}=169), 2.4 \%$ as Middle Eastern North African (MENA) or Southwest Asian North African (SWANA; $n=24), 1.6 \%$ as African American/Black ( $\mathrm{n}=16)$, and $11.2 \%$ as Other Ethnic ( $\mathrm{n}=113$; e.g., bi-or-multi ethnic, American Indian/Alaska Native, Native Hawaiian, Pacific Islander, etc.). Due to small n's for MENA/SWANA, African American/Black, and the ethnic groups comprised within Other Ethnic, statistical comparisons were only made for Asian, Hispanic/Latine, and NHW groups. Thus, the final sample totaled 855 participants.

\subsection{Materials}

Demographics Questionnaire (DEMO): A 41-item demographic questionnaire asked participants to identify their gender, ethnicity, age, perceptions of change between pre- and post-COVID 19 shelter-in-place mandates, and coping.

Generalized Anxiety Disorder Screener (GAD-7): The GAD-7 [23] is a seven-item self-report questionnaire used to measure anxiety severity. Higher scores indicate more severe symptom presentation. Responses up to 12 indicate mild anxiety symptom levels, scores around 17 equate to moderate anxiety symptom levels, and responses above 22 indicate severe anxiety symptom levels. Acceptable internal consistency $(\alpha=0.89)$ and convergent validity (r's with the Patient Health Questionnaire 2Depression, Rosenberg Self-Esteem Scale, Questionnaire on Life Satisfaction, and the Resilience Scale were 0.64, -0.43, -0.34, and -0.29 , respectively; all r's within $95 \% \mathrm{CI}$ range) were supported [23].

Perceived Stress Scale-10 (PSS-10): The PSS-10 [24] is a 10 item self-report questionnaire used to measure recognized pressure or nervous tension (i.e., low, moderate, or severe). Higher scores equate to greater perceived stress. Based on the scale, scores 10-23 indicate low stress, scores 24-36 equate to moderate stress, and scores 37-50 demonstrate high stress. The PSS10 has been found to be valid and reliable with Hispanic Americans [25]. Adequate internal consistency reliability for the PSS10 total and PSS-10 "Negative" subscale was demonstrated ( $\alpha$ 's=.82 \& .89, respectively). Significant correlations were reported between scores on the PSS-10 (total) and PSS-10 "Negative" subscale and scores on the Generalized Anxiety Disorder Screener or GAD-7 (r's=.64-.71) and the Patient Health Questionnaire-9 or PHQ-9 (measure of depression; r's=.56-.64), indicating acceptable convergent validity.

Perception of Academic Stress Scale (PASS): The PASS [26] is an 18 item self-report questionnaire used to measure perceived academic stress. For the current study, only items of the third subscale (i.e., stresses related to students' academic self-perceptions) were administered. A higher score represents a greater level of perceived academic stress. Scores from 6-14 were considered low academic stress, scores 15-22 showed moderate academic stress, and scores 23-30 indicated high perceived stress. Internal reliability for the PASS and for the Academic Self-Perceptions subscale ( $\alpha$ 's $=0.7 \& 0.5$, respectively) appeared modest but acceptable. Experts rated the PASS items as representative of academic stress and their overall agreement was $89 \%$, evidence of good content validity.

\subsection{Procedure}

This study was approved by CSUF's Institutional Review Board (IRB) and conducted in accordance with legal and ethical standards. Participants were given a weblink to the protocol on Qualtrics, an online survey program. After reading the informed 
consent, participants who continued, indicating consent, accessed the online protocol. Next, participants answered the DEMO, followed by the GAD-7, PSS-10, and PASS. After submitting their responses, participants viewed a debriefing "thanks" webpage that contained mental health resources along with links that provided more information on COVID-19.

\section{Results}

\subsection{Statistical analysis}

To test our predictions, we performed crosstabs, correlations, and multiple regression. All statistical analyses were performed on Statistical Package for Social Sciences, version 27 (SPSS v.27). The significance level cut-off was below .05. All statistical assumptions were tested and within acceptable criteria. There were no outliers.

\subsection{Academic engagement pre- and post-COVID-19}

Before COVID-19, most participants rated their level of academic engagement as high (56.2\%), with 27.9\% rating it as neutral, and $15.9 \%$ rating their level as low. Post COVID-19, academic engagement decreased with the majority of college students now reporting low academic engagement (61.2\%). 16.5\% of students rated their level of engagement as neutral, and $22.3 \%$ of students rated their academic engagement as high (see FIG. 1).

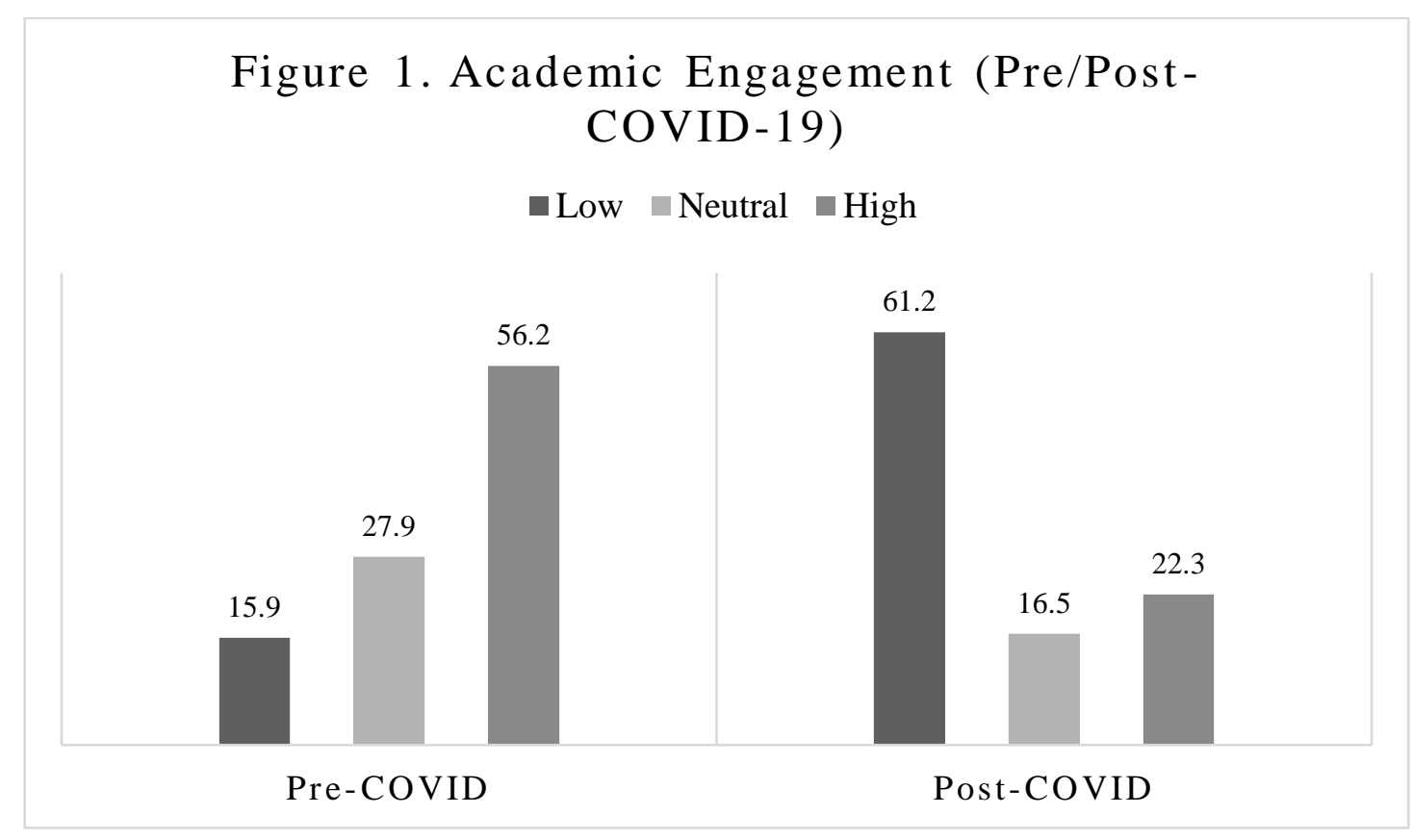

FIG. 1. Academic Engagement (Pre/Post- COVID-19).

Note. Values at the top of each bar represent the percentage of participants who endorsed that rating option.

\subsection{In-person classes to remote instruction transition rating}

$54.8 \%$ of Asians ( $n=92), 66.9 \%$ of Hispanic/Latine $(n=291)$, and $62.7 \%$ of NHW college students $(n=143)$ perceived the transition to remote instruction as difficult (see FIG. 2). 


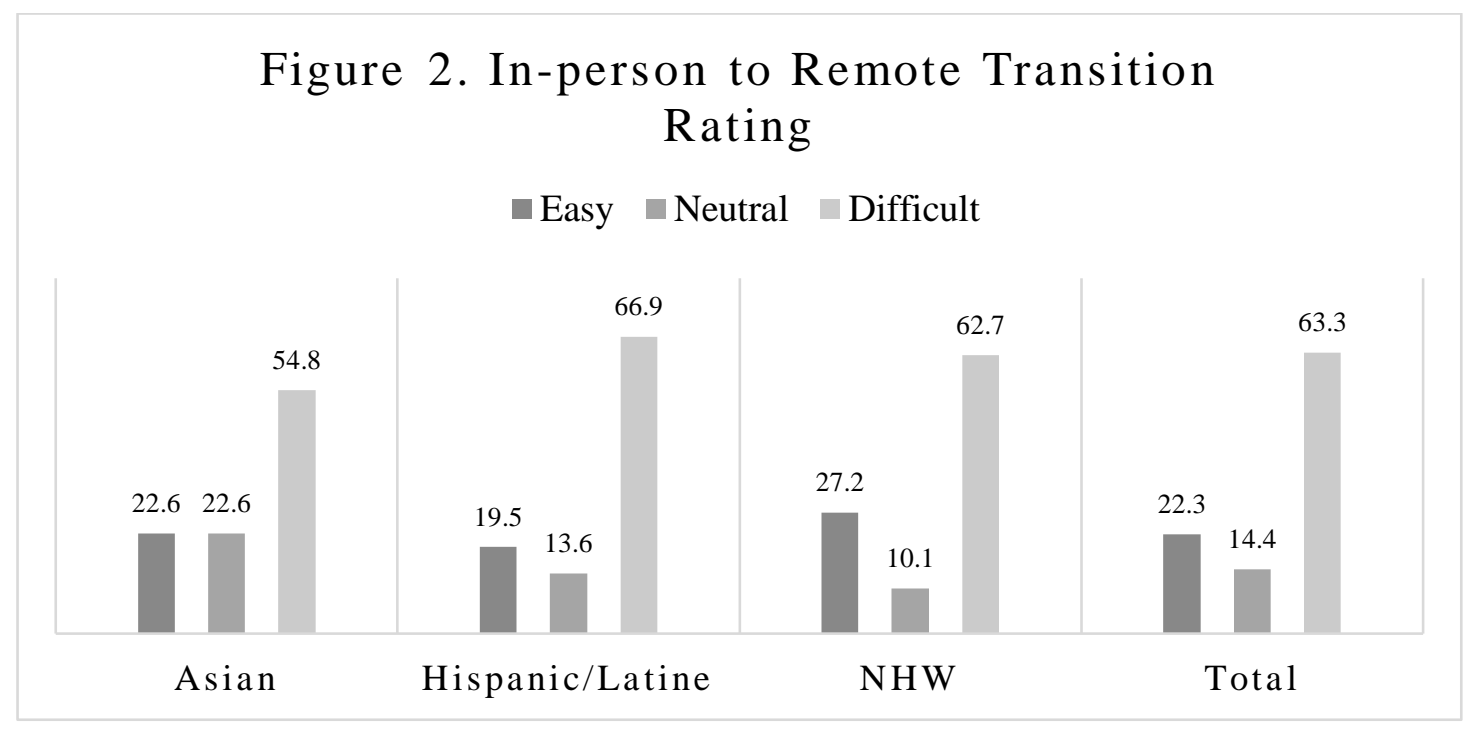

FIG. 2. In-person to Remote Transition Rating.

Note. Values at the top of each bar represent the percentage of participants in each ethnic group who endorsed that rating option.

NHW: Non-Hispanic White.

\subsection{General anxiety post COVID-19}

On the GAD-7, Asian college students averaged 17.43 ( $\mathrm{SD}=6.00)$, Hispanic/Latine students averaged 17.54 (SD=6.22), and NHW students averaged 16.56 ( $\mathrm{SD}=5.79$ ). Overall, most college students, regardless of ethnicity, reported moderate to high levels of anxiety: Asian 75.5\% ( $\mathrm{n}=126$ ); Hispanic/Latine 75.6\% ( $\mathrm{n}=339$ ); non-Hispanic White 72.0\% (NHW; $\mathrm{n}=170$; see FIG. $3)$.

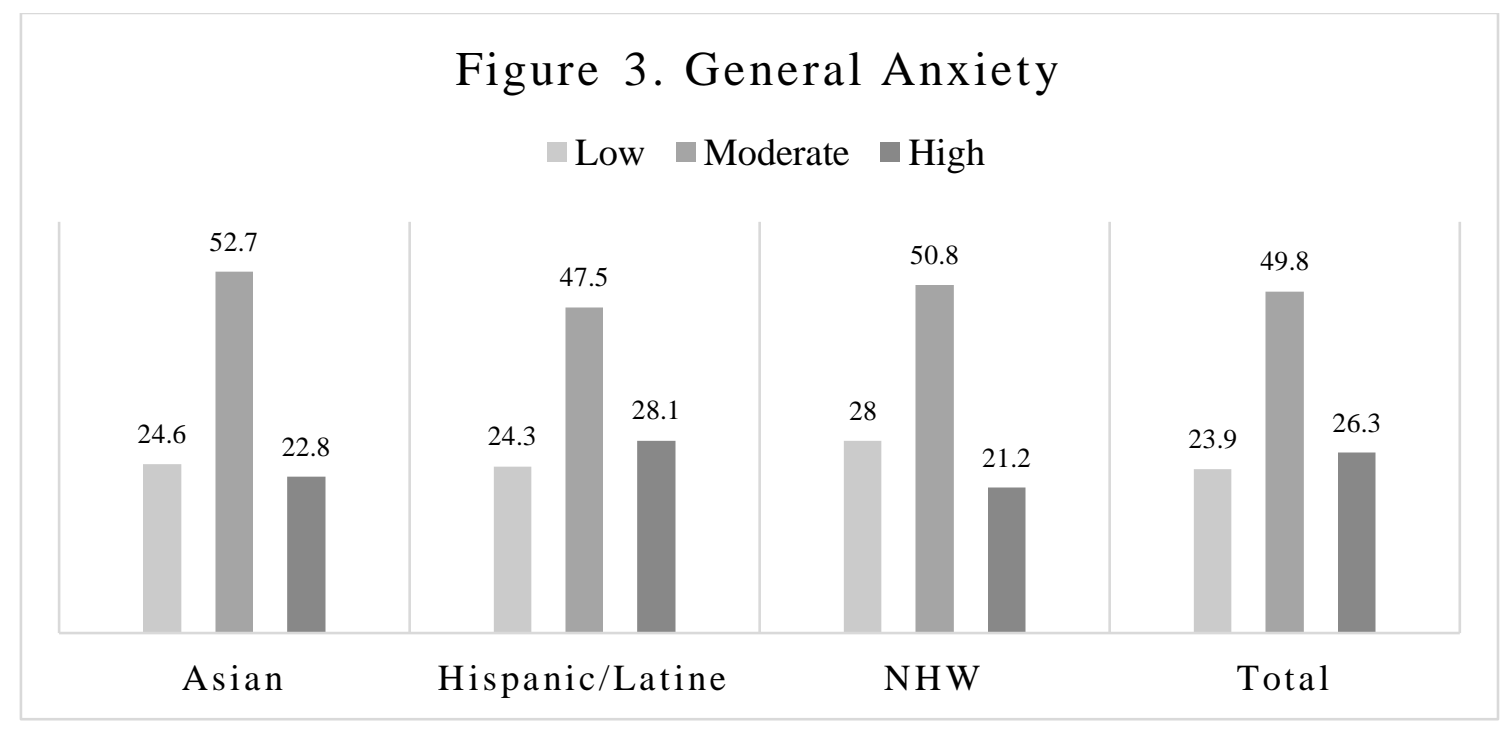

FIG. 3. General Anxiety.

Note. Values at the top of each bar represent the percentage of participants in each ethnic group who endorsed that rating option. NHW: Non-Hispanic White. 


\subsection{Perceived stress post COVID-19}

Hispanic/Latine college students had an average of 32.28 ( $\mathrm{SD}=7.86$ ) on the PSS-10, Asian students had an average of 32.07 ( $\mathrm{SD}=6.93$ ), and NHW students had an average of 30.74 ( $\mathrm{SD}=7.90)$. Overall, the majority of all ethnic groups reported moderate to high levels of stress: Asian 88.5\% ( $\mathrm{n}=146$ ); Hispanic/Latine 86.4\% ( $\mathrm{n}=381)$; NHW 81.3\% ( $\mathrm{n}=192$; see FIG. 4).

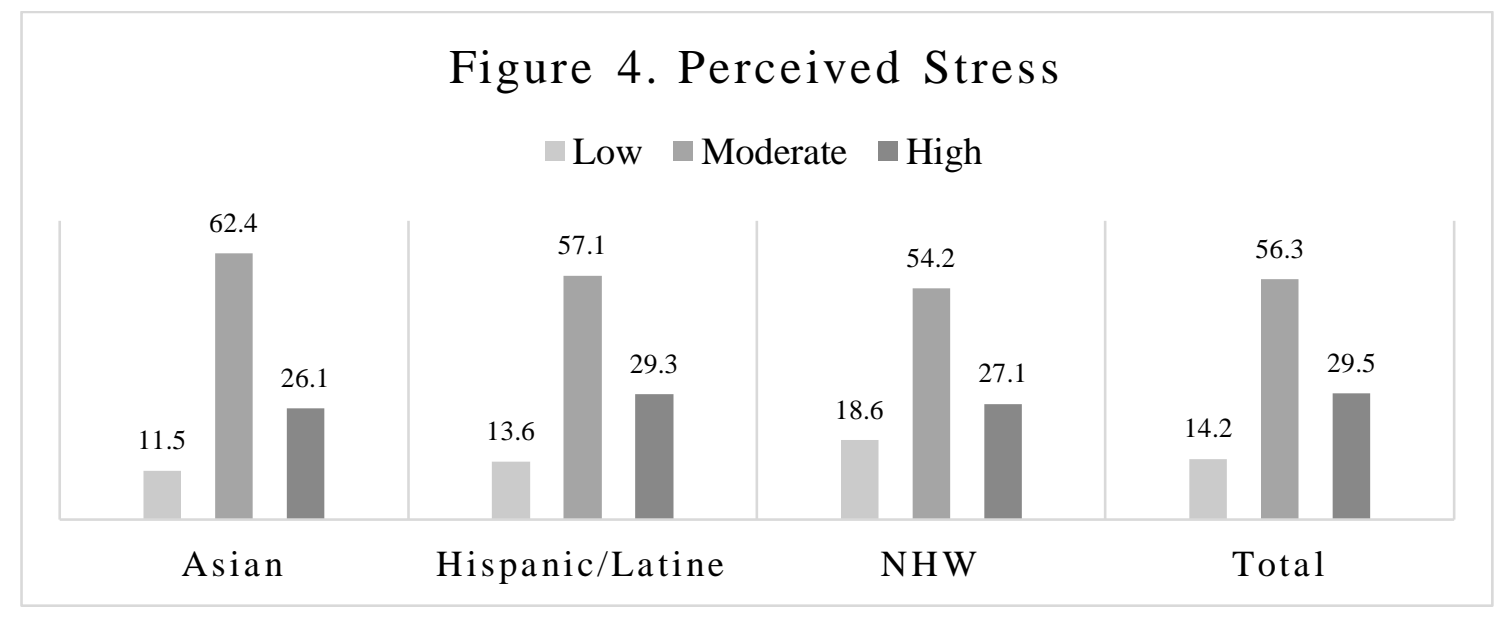

FIG. 4. Perceived Stress.

Note. Values at the top of each bar represent the percentage of participants in each ethnic group who endorsed that rating option. NHW: Non-Hispanic White.

\subsection{Perceived academic stress post COVID-19}

Asian students had an average of 18.11 ( $\mathrm{SD}=5.14$ ), Hispanic/Latine students had an average of 17.38 ( $\mathrm{SD}=5.12$ ), and NHW students had an average of 14.92 ( $\mathrm{SD}=5.42$ ). Overall, the majority of students reported moderate to high academic stress: Asian 76.8\% ( $\mathrm{n}=129$ ); Hispanic/Latine 72.8\% ( $\mathrm{n}=326$ ); NHW 52.3\% (n=123; see FIG. 5).

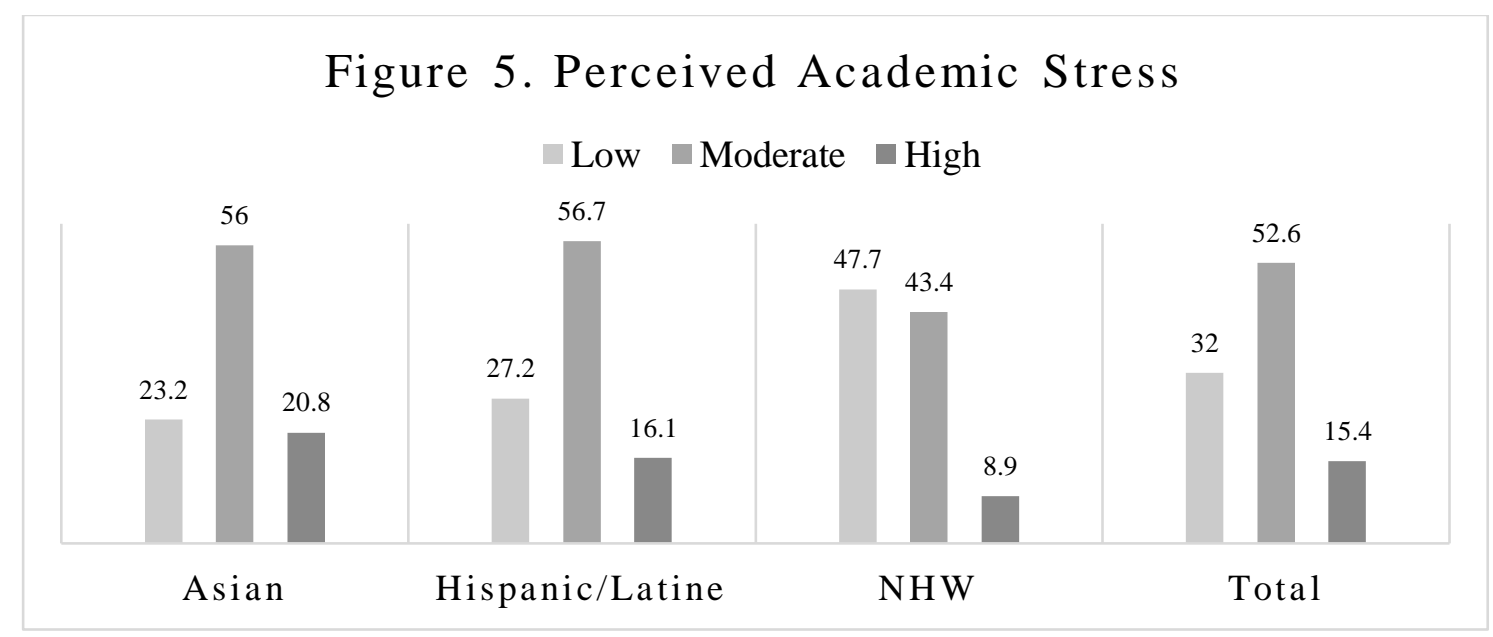

FIG. 5. Perceived Academic Stress.

Note. Values at the top of each bar represent the percentage of participants in each ethnic group who endorsed that rating option. NHW: Non-Hispanic White. 


\subsection{Healthy coping post COVID-19}

In terms of healthy coping activities, NHW college students were most likely to exercise (23\%; $=53)$, Asian students most commonly watched television or played video games ( $30 \%$; $=51$ ), while Hispanic/Latine students engaged in using social media (20\%; $\mathrm{n}=90)$ and watching television or playing video games (20\%; $\mathrm{n}=90$; see FIG. 6).

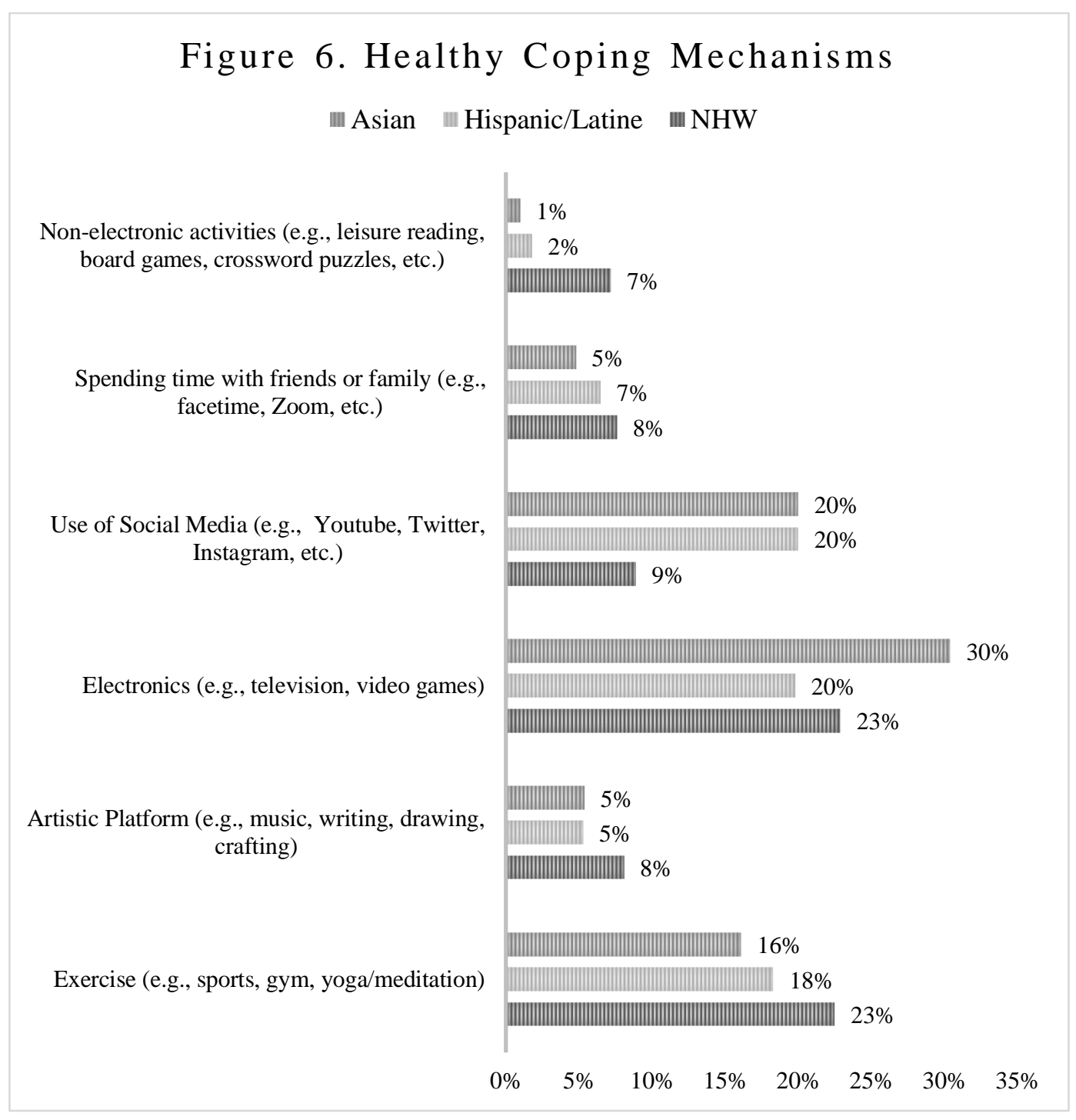

FIG. 6. Healthy coping mechanisms.

Note. NHW: Non-Hispanic White.

\subsection{Unhealthy coping post COVID-19}

The top five maladaptive coping mechanisms were: excessive drinking (16.9\%), excessive use of marijuana or other synthetic cannabinoids (13.7\%), engaging in acts of aggression or violence (8.5\%), excessive use of prescription medications (7.6\%), and driving recklessly (4.7\%). Excessive alcohol consumption was the most commonly reported unhealthy coping activity of non-Hispanic White (23\%; n=55) and Hispanic/Latine (19\%; n=84) students. For Asian students, excessive use of marijuana $(9 \% ; n=15)$ was the most endorsed (see FIG. 7). 


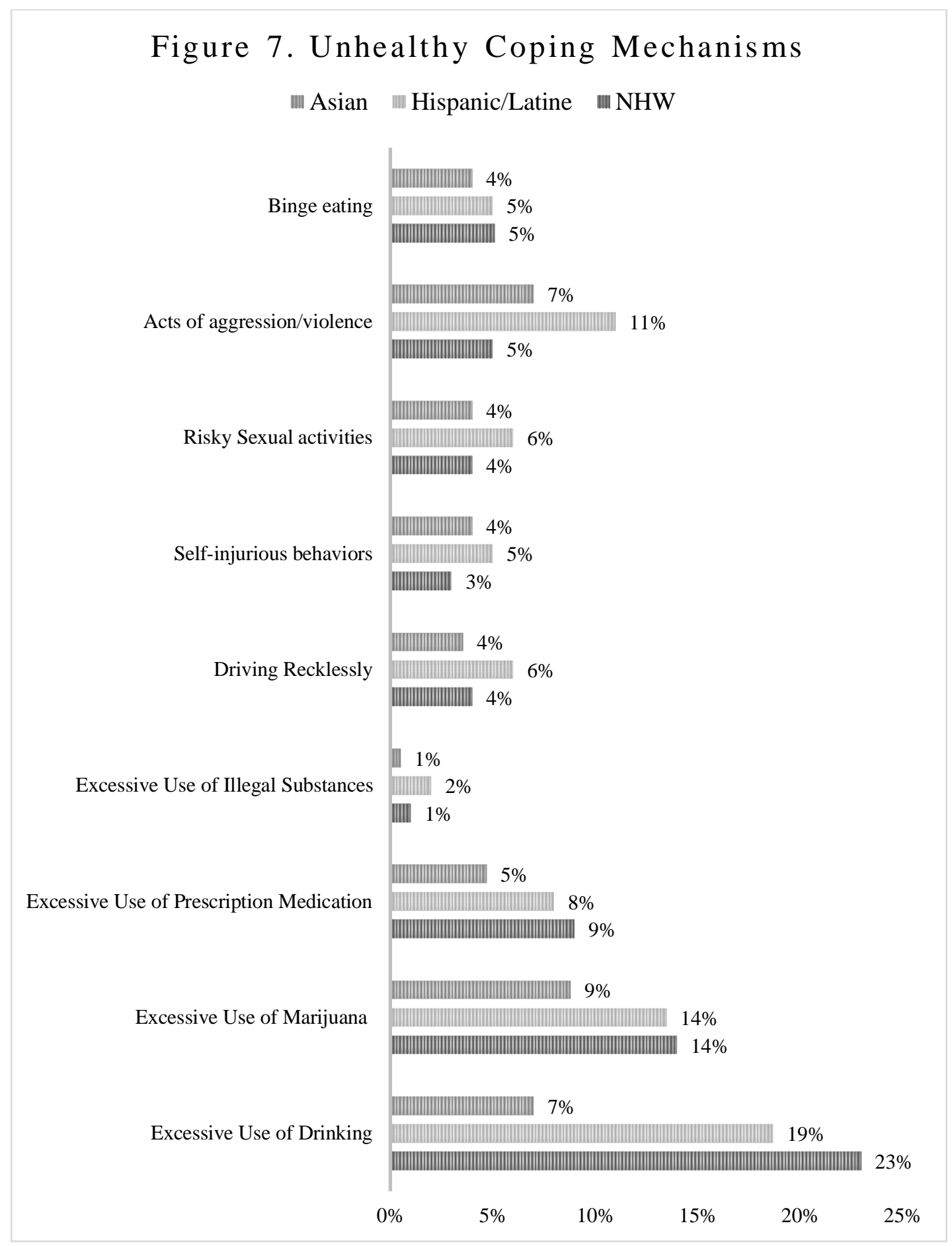

FIG. 7. Unhealthy Coping Mechanisms.

Note. NHW: Non-Hispanic White.

\subsection{Correlational analyses}

Intercorrelations were conducted among scale totals (see TABLE 1). Academic engagement was significantly negatively correlated with transition rating $(\mathrm{r}=-.29)$, general anxiety $(\mathrm{r}=-.15)$, and both stress scales. Transition rating was significantly positively correlated with general anxiety ( $\mathrm{r}=.27)$, and both stress scales. 
TABLE 1. Intercorrelations of Scale Totals.

\begin{tabular}{|c|c|c|c|c|c|}
\hline & $\begin{array}{c}\text { Academic } \\
\text { Engagement }\end{array}$ & $\begin{array}{c}\text { Transition } \\
\text { Rating }\end{array}$ & $\begin{array}{c}\text { General } \\
\text { Anxiety Scale }\end{array}$ & $\begin{array}{c}\text { Perceived } \\
\text { Stress Scale }\end{array}$ & $\begin{array}{c}\text { Perceived Academic } \\
\text { Stress Scale }\end{array}$ \\
\hline $\begin{array}{c}\text { Academic } \\
\text { Engagement }\end{array}$ & 1.0 & & & & \\
\hline $\begin{array}{c}\text { Transition Rating } \\
\text { General Anxiety } \\
\text { Scale }\end{array}$ & $-.29^{* * *}$ & 1.0 & & & \\
\hline $\begin{array}{c}\text { Perceived Stress } \\
\text { Scale }\end{array}$ & $-.26^{* * * * *}$ & $.27^{* * *}$ & 1.0 & & \\
\hline $\begin{array}{c}\text { Perceived Academic } \\
\text { Stress Scale }\end{array}$ & $-.27^{* * * *}$ & $.35^{* * * * *}$ & $.75^{* * * *}$ & $.58^{* * * * *}$ & \\
\hline
\end{tabular}

\subsection{Regression analyses}

Multiple linear regression analyses were conducted to assess whether transition rating and academic engagement predicted anxiety, stress, and academic stress. All assumptions (e.g., normality, homoscedasticity, multicollinearity, and outliers) were tested and met. The results of the multiple linear regression model predicting anxiety were significant, $F(2,824)=34.95$, $p<.001$, $\mathrm{R}^{2}=0.08$, indicating that approximately $8 \%$ of the variance in anxiety is explainable by transition rating and academic engagement. Transition rating significantly predicted anxiety indicating that moving from the easy to difficult category of transition rating increased the mean value of anxiety by 1.73 units on average. Additionally, academic engagement significantly predicted anxiety suggesting that moving from the low to high category of academic engagement decreased the mean value of anxiety by 1.20 units on average (see TABLE 2 ).

TABLE 2. Results for Multiple Linear Regression with Transition Rating and Academic Engagement Predicting Anxiety.

\begin{tabular}{|c|c|c|c|c|c|c|}
\hline Variable & $\boldsymbol{B}$ & $\boldsymbol{S E}$ & $\mathbf{9 5 \%}$ CI & $\boldsymbol{\beta}$ & $\boldsymbol{t}$ & $\boldsymbol{p}$ \\
\hline (Intercept) & 13.88 & 0.46 & {$[12.20,15.56]$} & 0.00 & 16.20 & 0.001 \\
& & & & & & \\
\hline Transition rating & 1.73 & 0.25 & {$[1.28,2.28]$} & 0.27 & 6.95 & 0.001 \\
\hline Academic engagement & -1.2 & 0.25 & {$[-1.09,-.08]$} & -0.08 & -2.29 & 0.022 \\
& & & & & & \\
\hline
\end{tabular}

The results of the multiple linear regression model predicting perceived stress were significant, $F(2,815)=69.30$, $p<.001$, $\mathrm{R}^{2}=0.15$, indicating that approximately $15 \%$ of the variance in stress is explained by transition rating and academic engagement. 
www.yumedtext.com | December-2020 | ISSN: 2582-3264 | https://dx.doi.org/10.46527/2582-3264.128

Transition rating significantly predicted reported stress, suggesting that moving from the easy to difficult transition rating category increased the mean value of stress by 2.73 units on average. Academic engagement significantly predicted stress indicating that moving from the low to high rating of academic engagement decreased the mean value of stress by 1.62 units on average (see TABLE 3).

TABLE 3. Results for Multiple Linear Regression with Transition Rating and Academic Engagement Predicting Stress.

\begin{tabular}{|c|c|c|c|c|c|c|}
\hline Variable & $\boldsymbol{B}$ & $\boldsymbol{S E}$ & $\mathbf{9 5 \%}$ CI & $\boldsymbol{\beta}$ & $\boldsymbol{t}$ & $\boldsymbol{p}$ \\
\hline (Intercept) & 27.94 & 0.85 & {$[25.87,30.20]$} & 0.00 & 26.48 & .001 \\
& & & & & & \\
\hline Transition rating & 2.73 & 0.32 & {$[2.11,3.34]$} & 0.30 & 8.69 & .001 \\
\hline Academic engagement & -1.62 & 0.31 & {$[-2.24,-.99]$} & -0.17 & -5.09 & .001 \\
\end{tabular}

Lastly, the results of the multiple linear regression model predicting perceived academic stress were significant, $\mathrm{F}(2,824)=70.86, \mathrm{p}<.001, \mathrm{R}^{2}=0.15$, indicating that approximately $15 \%$ of the variance in academic stress is explained by transition rating and academic engagement. Transition difficulty significantly predicted academic stress. Moving from easy to difficult transition ratings increased the mean value of academic stress by 1.83 units on average. Academic engagement also significantly predicted academic stress indicating that moving from the low to high category of academic engagement decreased the mean value of academic stress by 1.20 units on average (see TABLE 4).

TABLE 4. Results for Multiple Linear Regression with Transition Rating and Academic Engagement Predicting Academic Stress.

\begin{tabular}{|c|c|c|c|c|c|c|}
\hline Variable & $\boldsymbol{B}$ & $\boldsymbol{S E}$ & $\mathbf{9 5 \%}$ CI & $\boldsymbol{\beta}$ & $\boldsymbol{t}$ & $\boldsymbol{p}$ \\
\hline (Intercept) & 11.06 & 0.58 & {$[9.92,12.20]$} & 0.00 & 20.04 & .001 \\
& & & & & & \\
\hline Transition rating & 1.83 & 0.22 & {$[1.39,2.24]$} & 0.34 & 9.11 & .001 \\
\hline Academic engagement & -1.20 & 0.21 & {$[-1.65,-.77]$} & -0.17 & -5.41 & .001 \\
\end{tabular}


www.yumedtext.com | December-2020 | ISSN: 2582-3264 | https://dx.doi.org/10.46527/2582-3264.128

\section{Discussion}

\subsection{COVID-19 \& academic experiences}

The current results clearly demonstrated that regardless of ethnicity, the impact of COVID-19 on academic adjustment and experiences was profound. The majority of participants reported that switching to remote from in-person learning was quite challenging (transition) and most students struggled on their own to remain focused on their classes and assignments (academic engagement).

\subsection{Transition from in person to virtual instruction}

Hispanic/Latine participants were the most likely to rate their adjustment to virtual classes as difficult (66.9\%), followed by NHW (62.7\%) and Asian (54.8\%) participants. Less than 20\% of Hispanic/Latine students rated their transition to online instruction as "Easy"; likewise, only $22.6 \%$ of Asian and $27.2 \%$ of NHW students rated their transition to online instruction as "Easy". The majority of participants clearly struggled in adapting to remote learning, with Hispanic/Latine participants most frequently experiencing difficulty with this modality.

\subsection{Academic engagement}

Prior to the COVID-19 mandated switch to remote instruction, only $15.9 \%$ of the sample rated their level of academic engagement as low. After the change to virtual classes, $61.2 \%$ of participants endorsed low academic engagement. Regardless of ethnicity, after switching to online instruction, the percentage of students who indicated low academic engagement almost quadrupled. Perhaps losing the routine of attending classes in person, meeting peers on campus to work on projects or to study together, spending time between classes dropping in on instructor office hours, preparing for their next class, or simply chatting with classmates - seemingly minor events that comprise one's academic schedule - represented such a significant loss of structure that many participants were challenged in developing a new academic schedule without these markers. Given that most participants were virtually attending classes or studying from their respective homes, their physical environment may have been far less conducive for learning and working on academic tasks than the campus/classroom. Also, issues related to online access necessary for academic work may have been other potential barriers to sustaining academic engagement and productivity.

\subsection{COVID-19 and anxiety}

Hispanic/Latine and Asian participants reported slightly higher general anxiety compared to their NHW peers. However, the majority of students in all three ethnic groups (Hispanic/Latine 75.6\%, Asian 75.5\%, NHW 72.0\%) endorsed moderate to high levels of anxiety two to three months after the COVID-19 mandated switch to virtual classes. The pre-coronavirus prevalence estimate of GAD in the US college population was 31\% [3]. Following the onset of the COVID-19 pandemic, the self-reported rate of general anxiety in the present sample was more than double the pre-COVID-19 national collegiate rate. This is consistent with mental illness increases in the US general population [27] across the same time period.

\subsection{COVID-19 and stress}

Like the findings for anxiety, Hispanic/Latine and Asian participants endorsed slightly higher levels of perceived general and academic stress than their NHW counterparts. However, all three ethnic groups reported moderate to high levels of general 
www.yumedtext.com | December-2020 | ISSN: 2582-3264 | https://dx.doi.org/10.46527/2582-3264.128

stress. Asian and Hispanic/Latine participants also endorsed moderate to high academic stress levels while just over half of the NHW participants indicated moderate to high academic stress.

\subsection{COVID-19 and general stress}

Regardless of ethnicity, the vast majority of respondents described experiencing significant stress. The percentages of each group reporting moderate to high stress levels were as follows: Asian (88.5\%), Hispanic/Latine (86.4\%), and NHW (81.3\%). While all three groups grappled with multiple stressors, Hispanic/Latine and Asian students were more likely to do so in contrast to their NHW peers.

\subsection{COVID-19 and academic stress}

In terms of perceived academic stress after the onset of-COVID-19 mandates, a greater proportion of students of color v. NHW students indicated higher levels of academic stress. While $76.8 \%$ of Asian participants and $72.8 \%$ of Hispanic/Latine participants rated their academic stress as moderate or high, just over half (52.3\%) of NHW participants perceived their academic stress as moderate to high. These findings suggest that COVID-19, while negatively affecting most participants' academic experiences, had a greater impact on students of color.

\subsection{COVID-19 coping}

For the most part, participants reported dealing with the myriad of challenges posed by COVID-19 in a generally positive manner. Some participants admitted to struggling to cope with pandemic life and engaged in self-destructive behaviors including substance abuse, aggressiveness, and binge eating.

\subsection{COVID-19 healthy coping}

Students endorsed exercising, watching television, playing video games, and using social media as their top selections for nonharmful ways to cope with their stress. Watching television/Netflix or playing video games were the top (healthy) coping activities chosen by Asian participants (30\%), while NHW participants were more likely to exercise (23\%). Hispanic/Latine participants were equally split when it came to healthy coping options, with $20 \%$ preferring to watch television/Netflix or play video games and $20 \%$ engaging with social media.

\subsection{COVID-19 unhealthy coping}

In terms of unhealthy means of coping, participants were most likely to (excessively) drink alcohol or smoke marijuana or synthetic cannabinoids. Other potentially harmful coping options included engaging in aggressive or violent acts, excessive use of prescription medications, reckless driving, and engaging in unprotected sexual intercourse. The most commonly endorsed unhealthy coping for Asians was excessive use of marijuana/synthetic cannabinoids (9\%), while the top method for both NHW (23\%) and Hispanic/Latine (19\%) participants was excessive use of alcohol.

\subsection{Academic predictors of anxiety and stress}

Both transition to online instruction and academic engagement were found to be significantly related to anxiety, general stress, and academic stress ratings. Participants who reported difficulty in adjusting to online instruction from in-person classes were more likely to endorse higher levels of anxiety, stress, and academic stress than those who navigated the transition with ease. 
www.yumedtext.com | December-2020 | ISSN: 2582-3264 | https://dx.doi.org/10.46527/2582-3264.128

Participants who endorsed low academic engagement following the onset of COVID-19 mandates were more likely to describe their anxiety, general stress, and academic stress as elevated than those who indicated high academic engagement.

Transition to virtual learning and academic engagement were significant predictors of anxiety, general stress, and academic stress. As a model, they accounted for $8 \%$ of the variance for anxiety and $15 \%$ of the variance for general stress and academic stress, respectively. These findings suggest that the academic experiences of participants were influential factors of anxiety and stress. Given that participants of color, compared to NHW participants, were more likely to report difficulty with adjusting to remote instruction and low post-COVID-19 academic engagement, as well as elevated levels of anxiety, general stress, and academic stress, it appears critical to provide these individuals with both academic and mental health resources.

\section{Study Limitations}

There are several limitations to the present study. First, it is a descriptive, cross-sectional study so that causal attributions cannot be drawn. A longitudinal investigation will be beneficial in better understanding the effects of academic experiences on subsequent anxiety and stress reactions of college students. The sample is small and drawn from one university. It is uncertain whether results are representative and can be generalized to US college students as a whole. Participants voluntarily completed the online survey with no material incentive; thus, sampling bias may have occurred.

The data were self-report in nature and there may be issues related to memory or social desirability. Respondents completed close-ended items and were not interviewed nor given an opportunity to expand on their answers. Future investigations may consider the value of including open-ended questions or interviews so that qualitative as well as quantitative analyses can be performed. Such data may provide insight and clarity into understanding the complexities involved in college students' mental health and coping with academic demands and life in general during the COVID-19 pandemic.

\section{Conclusions}

The majority of Asian and Hispanic/Latine participants, along with their non-Hispanic White peers, reported moderate to high anxiety, general stress, and academic stress post-onset of COVID-19 changes. Hispanic/Latine and Asian participants typically endorsed greater difficulty adjusting to remote instruction and virtual college life than NHW participants. Similarly, participants of color (v. NHW participants) were more likely to report higher levels of anxiety, stress, and academic stress. Perceived academic engagement and transition from in-person to remote instruction predicted anxiety, stress, and academic stress. On a positive note, participants mostly endorsed using healthy coping techniques, rather than unhealthy coping activities.

Although being more psychologically vulnerable due to the impact of COVID-19, college students of color may be less likely than their White counterparts to report mental health issues or to seek out professional intervention. Consequently, academic performance and retention may be undermined. College health professionals should provide culturally sensitive outreach and proactively engage ethnically diverse students in prevention and support services. Additional academic resources should be made available, particularly to students of color such as those identifying as Hispanic/Latine and Asian, as COVID-19 academic experiences and perceptions appear to be strongly connected to anxiety and stress levels of college students. 
www.yumedtext.com | December-2020 | ISSN: 2582-3264 | https://dx.doi.org/10.46527/2582-3264.128

\section{Acknowledgements}

The authors would like to thank the following for their assistance and support of this study:

Melinda Blackman, Ph.D., Chair, Psychology Department, College of Humanities \& Social Sciences, California State University, Fullerton (CSUF), USA.

Sue Maddox, Administrative Analyst/Specialist, Psychology Department, College of Humanities \& Social Sciences, CSUF, USA.

Eriko Self, Ph.D., (Former) Department Chair \& Professor, Psychology Department, College of Humanities \& Social Sciences, CSUF, USA.

Su Swarat, Ph.D., Associate Vice President for Institutional Effectiveness \& Accreditation Liaison Officer, Academic Affairs, CSUF, USA.

Afshin Karimi, Sr. Research Associate \& IR Lead, Office of Assessment \& Institutional Effectiveness, Academic Affairs, CSUF, USA.

CSUF Student Participants for their willingness, time, and openness in voluntarily completing this survey.

\section{REFERENCES}

1. Kane W. Anxiety 'epidemic' brewing on college campuses, researchers find. Berkeley News; 2019, April 18. [https://news.berkeley.edu/2019/04/18/anxiety-epidemic-brewing-on-college-campuses-researchers-find/]

2. American College Health Association. Reference Group Data Report; 2018. [https://www.acha.org/documents/ncha/NCHA-II_Fall_2018_Reference_Group_Data_Report.pdf]

3. Healthy Minds Network. The Healthy Minds Study. 2019. [https://healthymindsnetwork.org/wpcontent/uploads/2019/09/HMS_national-2018-19.pdf]

4. Center for Collegiate Mental Health. 2019 Annual Report (Publication No. STA 20-244), [https://ccmh.psu.edu/assets/docs/2019-CCMH-Annual-Report_3.17.20.pdf]

5. Asimov N. Rising anxiety on campuses linked to finances, phones in UC Berkeley study. SFChronicle.com; 2019, April 22. [https://www.sfchronicle.com/bayarea/article/Rising-anxiety-on-campuses-linked-to-finances13781698.php]

6. Lederman D. Presidents' biggest COVID-19 worries? Low-income students and colleges' financial strain. Inside Higher Ed; 2020, April 27. [https://www.insidehighered.com/news/survey/presidents-biggest-covid-19-worries-lowincome-students-and-colleges-financial-strain]

7. Lederman D. College presidents fear financial -- and human -- toll of coronavirus on their campuses. Inside Higher Ed; 2020, March 27. [https://www.insidehighered.com/news/survey/college-presidents-fear-financial-and-humantoll-coronavirus-their-campuses]

8. American College Health Association \& Healthy Minds Network. The impact of COVID-19 on college student wellbeing; 2020. [https://www.acha.org/documents/ncha/Healthy_Minds_NCHA_COVID_Survey_Report_FINAL.pdf]

9. Active Minds. COVID-19 Impact on college student mental health; 2020, April. [https://www.activeminds.org/wpcontent/uploads/2020/04/Student-Survey-Infographic.pdf]. 
www.yumedtext.com | December-2020 | ISSN: 2582-3264 | https://dx.doi.org/10.46527/2582-3264.128

10. Huckins JF, daSilva A, Wang W, et al. Mental Health and Behavior of College Students During the Early Phases of the COVID-19 Pandemic: Longitudinal Smartphone and Ecological Momentary Assessment Study. J Med Internet Res. 2020;22(6):e20185.

11. TimelyMD. College students' mental health continues to suffer from COVID-19, new survey by TimelyMD finds.TimelyMD; 2020, July 23. [https:/www.timely.md/college-students-mental-health-continues-to-suffer-fromcovid-19-new-survey-by-timelymd-finds/].

12. Li HY, Cao H, Leung DYP, et al. The psychological impacts of a COVID-19 outbreak on college students in China: A longitudinal study. Int J Envir Res Pub Health. 2020;17(11):3933.

13. Pragholapati A. COVID-19 impact on students. [Internet]. EdArXiv;2020. Available from: edarxiv.org/895ed doi:10.17605/OSF.IO/NUYJ9

14. Odriozola-González P, Planchuelo-Gómez Á, Irurtia-Muñiz MJ, et al. Psychological effects of the COVID-19 outbreak and lockdown among students and workers of a Spanish university. Psychiatry Res. 2020;290:113108.

15. Savitsky B, Findling Y, Ereli A, et al. Anxiety and coping strategies among nursing students during the covid-19 pandemic. Nurse Educ Pract. 2020;46:102809.

16. Husky MM, Kovess-Masfety V, Swendsen JD. Stress and anxiety among university students in France during Covid19 mandatory confinement. Compr Psychiatry. 2020;102:152191.

17. Naser AY, Dahmash EZ, Al-Rousan R, et al. Mental health status of the general population, healthcare professionals, and university students during 2019 coronavirus disease outbreak in Jordan: A cross - sectional study. Brain Behav. 2020;10(8):e01730.

18. Pew Research Center. U.S. Hispanic population surpasses 60 million in 2019, but growth has slowed. Pew Research Center; 2020. [https://www.pewresearch.org/fact-tank/2020/07/07/u-s-hispanic-population-surpassed-60-million-in2019-but-growth-has-slowed/]

19. Willott L. Average survey response rate-What you need to know. Customer Thermometer; 2019, July 12. [https://www.customerthermometer.com/customer-surveys/average-survey-response rate/\#: :text=A\%20survey\%20response\%20rate\%20of,range\%20are\%20far\%20more\%20typical.]

20. Lindemann N. What's the average survey response rate? Survey/Anyplace; 2019, August 8. [https://surveyanyplace.com/average-survey-response-rate/].

21. Fan W, Yan Z. Factors affecting response rates of the web survey: A systematic review. Comput Human Behav. 2010;26(2):132-9.

22. Manfreda KL, Bosnjak M, Berzelak J, et al. Web surveys versus other survey modes: a meta-analysis comparing response rates. Int J Market Res. 2008;50(1):79-104.

23. Löwe B, Decker O, Müller S, et al. Validation and standardization of the Generalized Anxiety Disorder Screener (GAD-7) in the general population. Med Care. 2008;46(3):266-74.

24. Cohen S, Williamson G. Perceived stress in a probability sample of the United States In: Spacapam S , Oskamp S, editors. The Social Psychology of Health. Newbury Park, CA: Sage, USA; 1988.

25. Baik SH, Fox RS, Mills SD, et al. Reliability and validity of the Perceived Stress Scale-10 in Hispanic Americans with English or Spanish language preference. J Health Psychol. 2019;24(5):628-39.

26. Bedewy D, Gabriel A. Examining perceptions of academic stress and its sources among university students: The Perception of Academic Stress Scale. Health Psychol Open. 2015;2(2):2055102915596714. 
www.yumedtext.com | December-2020 | ISSN: 2582-3264 | https://dx.doi.org/10.46527/2582-3264.128

27. Czeisler MÉ, Lane RI, Petrosky E, et al. Mental health, substance use, and suicidal ideation during the COVID-19 pandemic - United States, June 24-30, 2020. MMWR Morb Mortal Wkly Rep. 2020;69:1049-57. 